\title{
Successful ageing in adversity: the LASER-AD longitudinal study
}

\author{
G Livingston, ${ }^{1}$ C Cooper, ${ }^{1}$ J Woods, ${ }^{1}$ A Milne, ${ }^{3}$ C Katona ${ }^{2}$
}

\begin{abstract}
${ }^{1}$ Department of Mental Health Sciences, University College London, Archway Campus, Highgate Hill, London, UK; ${ }^{2}$ Kent Institute of Medicine and Health Sciences, University of Kent, Canterbury, Kent, UK; ${ }^{3}$ Tizard Centre, School of Social Policy, Sociology and Social Research, University of Kent, Canterbury, Kent, UK
\end{abstract}

Correspondence to: Professor G Livingston, Department of Mental Health

Sciences, University College London, Holborn Union Building, Archway Campus, Highgate Hill, London N19 5LW, UK g.livingston@ucl.ac.uk

Received 7 June 2007 Revised 3 August 2007 Accepted 5 September 2007

Published Online First 26 September 2007

\section{ABSTRACT}

Background: Most models of successful ageing do not allow for the possibility of living "successfully," despite some degree of cognitive or physical impairment. We reviewed the successful ageing and related quality of life literature to identify their potential predictors. We then tested our hypotheses that wellbeing in adversity would be predicted by mental health (anxiety and depression) and social factors rather than physical health and that it would be stable over time.

Method: We interviewed 224 people with Alzheimer's disease (AD) and their family carers, recruited to be representative of those living with $A D$ in the community. We re-interviewed 122 (73.1\% of eligible) participants 18 months later. Our main outcome measure was the perception of the person with $A D$ on their life as a whole. Results: Mean "wellbeing in adversity" scores did not change significantly over time $(t=0.23)$. Social relationships, subjective mental health, health perception, activities of daily living and baseline wellbeing in adversity were the significant correlates of wellbeing in adversity on univariate analysis. Only baseline wellbeing in adversity and mental health score were significant predictors in our regression analysis. In a well fitting structural equation model, less severe dementia and better health perception predicted fewer mental health problems and social relationships, but were not direct predictors of wellbeing in adversity at 18 months.

Conclusion: Successful ageing was common among a cohort of people with dementia. The most important predictors of this were mental health and social relationships, which fully mediated the relationship we found between health perception and wellbeing 18 months later.

Successful ageing may not only be about escaping illness but also of having a positive attitude towards one's life despite poor health. ${ }^{1}$ The literature tends to define "successful ageing" as the absence of physical and cognitive impairment, usually neglecting the possibility of positive adaptation or resilience in the face of health related adversity. ${ }^{1}$ It is not surprising then, that younger age is the most consistent predictor of successful ageing. ${ }^{1}$ Older adults commonly stress that social engagement and positive outlook towards life are more important than physical health status, but these are often not considered at all, or are not viewed as equal facets. ${ }^{1}$ Thus the dominant model of successful ageing is deficit based, and does not include older people who may be living "successfully", with some degree of impairment.

In contrast, quality of life (QoL) is a concept which generally encompasses "how good" a person's life is overall. It adopts a more inclusive, asset based approach to the notion of ageing "successfully", allowing for the possibility of resilience ${ }^{2}$ or successful ageing despite adversity. Many people with chronic conditions consistently rate their own quality of life highly. ${ }^{3}$ This "disability paradox" highlights the multidimensional nature of QoL, which encompasses emotional, social and psychological as well as health related domains. ${ }^{45}$ Nevertheless, traditional health related QoL models are likely to underestimate quality of life in people with significant health problems due to their emphasis on physical life domains. ${ }^{6}$ Thus although OoL scales include diverse domains which contribute to overall $\mathrm{OoL}$, their standardised structure means they are unlikely to capture the highly idiosyncratic, subjective nature of $\mathrm{QoL}^{78}$ and may exclude important group level variables (such as spirituality ${ }^{9}$.

Despite the cognitive and communication impairments associated with the condition, there is evidence that people with dementia, even at the severe stages, can give valid and consistent answers about QoL, ${ }^{10-12}$ which are not related to insight into their illness. ${ }^{24}$ In addition, proxy informants systematically rate the QoL of people with dementia as lower than those with the illness do themselves, ${ }^{24}$ and this may also apply to nondemented elderly people, at least with regards to certain QoL domains. ${ }^{13}$

People with Alzheimer's disease (AD) consistently rate their QoL highly ${ }^{14}{ }^{15}$ and equivalent to self-ratings by older people without dementia. ${ }^{2}{ }^{13}$ We reviewed the literature regarding associates of self-reported QoL in people with dementia in order to help us construct a model of factors predicting successful ageing in this population. The most consistent predictor was mental health (depres$\operatorname{sion}^{11} 1^{14-21}$ and anxiety, ${ }^{11} 1720$ or both ${ }^{22}$ ). We also found that functional status ${ }^{18} 1923$ (which reflects physical health as well as cognitive severity), neuropsychiatric symptoms, ${ }^{20} 24$ global rating of dementia severity ${ }^{1725}$ and education ${ }^{14}{ }^{19}$ were associates in at least two studies. Severity of cognitive impairment (as measured by the Mini Mental State Examination) is consistently unrelated to self-reported OoL. $24811121416-182026 \mathrm{We}$ also included pain, ${ }^{21}$ physical health ${ }^{18} 19$ and social relationships in our study because although their relationship with QoL in people with dementia have not often been reported, they are strongly related to depression and to GoL in people with other illnesses. ${ }^{27}$

Single item ratings of health are consistent and strong predictors of morbidity and mortality, ${ }^{14}$ possibly because they reflect the individual, holistic nature of the rater's experience. In the current 
paper, we sought to determine the predictors of successful ageing, or wellbeing in adversity, in a cohort of people with $\mathrm{AD}$ using a self-rated, single item "life as a whole" measure. Our main hypothesis was that wellbeing in adversity over 18 months would be predicted by baseline mental health (anxiety and depression) and by social factors rather than global dementia severity, pain and physical illness (measured by prescribed). Our second hypothesis was that wellbeing in adversity reflects an individual's underlying resilience and would therefore be stable over time.

\section{METHODS}

This is part of a larger, naturalistic, longitudinal study of people with $\mathrm{AD}$, covering the London and the South East Region (LASER-AD study ${ }^{28}{ }^{29}$ ). The local research ethics committees gave ethics approval for the study. Participants were contacted through local psychiatric services, the voluntary sector, and nursing and residential care homes. They were prospectively recruited to be a representative sample of people with $\mathrm{AD}$ in terms of sex, living setting and severity of cognitive impairment in the community, the latter of which was categorised as follows: mild $30 \%$, moderate $40 \%$ and severe $30 \%{ }^{30}$ The inclusion criteria were a standardised diagnosis of dementia (American Psychiatric Association) and fulfilment of criteria for possible or probable $\mathrm{AD} .{ }^{31}$ Interviews were conducted at baseline and 18 months later.

\section{Data collection}

We obtained written informed consent for all carers. Where the care recipient (CR) lacked capacity to consent, we only proceeded if the carer was happy for us to do so and thought the CR would have agreed to participate if they could, and the $\mathrm{CR}$ assented. We terminated the interview if the interviewee became distressed or appeared to want to stop. Trained researchers collected all information but were blind to the hypothesis of the study reported here.

\section{Interview}

Trained researchers collected data including sociodemographic details with age and years of full time education. They also completed the following measures.

- Mini Mental State Examination $(\mathrm{MMSE})^{32}$ to measure cognitive impairment.

- Neuropsychiatric Inventory ${ }^{33}$ to measure 12 psychiatric symptoms (delusions, hallucinations, dysphoria, anxiety, elevated mood, sleep, appetite, irritability, aberrant motor behaviour, aggression/agitation, apathy and disinhibition). severity (score of 1-3) or as absent (score 0). The frequency score is multiplied by the severity score for each symptom and the sum of these creates a global score (maximum score $=144$ ).

- The Alzheimer's Disease Co-operative Study InventoryActivities of Daily Living $(\mathrm{ADL})^{34}$ is a 23 item questionnaire measuring functional status completed by the carer and scores range between 0 and 78. Higher scores indicate less impaired ADL functioning.

- Depressive illness in CRs was rated using the Cornell Scale for Depression in Dementia. ${ }^{35}$ Higher scores mean more depressive symptoms. patient's overall general health perception or number of drugs

\section{Setting and recruitment} Carers rate each symptom by frequency (score 1-4) and

- We used the global dementia severity item from the Clinician Interview Based Impression of Change Plus. ${ }^{36}$ Higher scores indicate greater severity.

- We used three subscales of the Health Status Questionnaire$12(\mathrm{HSO}-12)^{37} 38$ to measure mental health (anxiety and mood), subjective health perception (self report of health) and pain. Higher scores indicated less pain, and better subjective health and mental health.

- We administered the Quality of Life-Alzheimer's Disease Scale (QoL-AD) ${ }^{19}$ and included in the analysis: (1) a total social relationships score, summating three individual item scores measuring the participant's perception of their relationships with family, their closest relationship and friends, and (2) response to the single item rating of life as a whole to measure our main outcome of "wellbeing in adversity". Possible scores ranged from 1 (poor) to 4 (excellent).

\section{Analysis}

We used SPSS 14.0 and AMOS V.7.0 to analyse the data, and report two tailed tests throughout. We report frequencies, means (SD) and confidence intervals (CI), as appropriate, to describe the sociodemographic and other characteristics of the sample. We explored the relationship of the determinants derived from the literature, and MMSE as a measure of cognition, with "wellbeing in adversity." If the skewness statistic was $\geqslant 1$, we transformed the data. ${ }^{39}$ Although we had two measures of mental health (Cornell Scale for Depression in Dementia, HSQ-mental health) we report results of only HSO mental health, as it includes anxiety and depression, and was more highly correlated with the outcome measure. We performed a paired sample $t$ test between "wellbeing in adversity" at baseline and 18 months.

We then entered the factors which approached significance $(p<0.1)$ in bivariate analysis into a linear regression analysis to explore the independent predictors of "wellbeing in adversity,"

Table 1 Descriptives and correlates of baseline postulated determinants with "wellbeing in adversity" at 18 months

\begin{tabular}{|c|c|c|c|}
\hline & \multicolumn{2}{|c|}{ Descriptives } & \multirow{2}{*}{$\begin{array}{l}\text { Pearson's } \\
\text { correlation } \\
\text { coefficient } \dagger\end{array}$} \\
\hline & $\mathbf{n}$ & Mean (SD) & \\
\hline \multicolumn{4}{|l|}{ Physical health indicators } \\
\hline Activities of daily living functioning & 122 & $46.4(17.7)$ & $0.21 *$ \\
\hline Pain & 122 & $2.0(1.4)$ & 0.10 \\
\hline No medications prescribed & 122 & $3.6(2.4)$ & -0.07 \\
\hline Subjective health perception & 122 & $62.4(28.1)$ & $0.26 * *$ \\
\hline \multicolumn{4}{|l|}{ Mental health and social relationships } \\
\hline Mental health & 122 & $76.7(19.1)$ & $0.44^{* * *}$ \\
\hline Cornell score & 122 & $3.8(3.9)$ & $-0.20^{*}$ \\
\hline Social relationship & 111 & $10.2(1.8)$ & $0.31 * *$ \\
\hline \multicolumn{4}{|l|}{ Dementia variables } \\
\hline Neuropsychiatric Inventory Total & 122 & $15.6(14.1)$ & -0.04 \\
\hline Global dementia severity & 122 & $3.7(0.8)$ & 0.01 \\
\hline MMSE score & 122 & $16.7(6.9)$ & 0.04 \\
\hline \multicolumn{4}{|l|}{ Sociodemographic variables } \\
\hline Age & 122 & $80.4(7.3)$ & -0.15 \\
\hline Years of education & 111 & $9.4(1.5)$ & 0.08 \\
\hline \multicolumn{4}{|l|}{ Main outcome variable } \\
\hline Baseline wellbeing in adversity & 122 & $3.0(0.7)$ & $0.44^{* *}$ \\
\hline
\end{tabular}


cross sectionally and at 18 months. Finally, we used structural equation modelling (which allows path analysis) to confirm our hypothesis that wellbeing over 18 months would be directly predicted by mental health (anxiety and depression) and by social factors rather than global dementia severity and overall health. We used Baron and Kenny ${ }^{40}$ criteria to define mediation. These require, for example, that in order to conclude that an identified relationship between a physical health measure and wellbeing was mediated by mental health, that the physical health measure should be associated with mental health on univariate analyses, and that the univariate relationship between physical health and wellbeing is either no longer significant or reduced in magnitude by controlling for mental health in a regression with wellbeing as the dependent variable.

To obtain the most parsimonious model, we used the accepted criterion that associations between variables should be removed from the model if the rise in $\chi^{2}$ for the model (which tests the null hypothesis that the model could have occurred by chance) was smaller than the critical value for that association. $\chi^{2}$ test results should not be significant or the model may have occurred by chance. We report the Normed Fit Index (NFI; a measure from 0 to 1 (perfect fit) of the extent to which the model fits the data), $\chi^{2}$ test for the final model and unstandardised regression estimates for each association. NFI $\geqslant 0.9$ is generally accepted as evidence of a well fitting model. ${ }^{41}$

\section{RESULTS}

\section{Baseline participants}

We interviewed 224 people with $\mathrm{AD}$ at baseline of whom 160 $(71.4 \%)$ were women. Their ages ranged from 55 to 98 years (mean 81.0 (SD 7.4)). MMSE scores ranged from 0 to 29 (mean 14.7 (SD 8.3)). Fifty-three (23.7\%) people lived alone, and 73 $(32.6 \%)$ in $24 \mathrm{~h}$ care. Mean years of education was 9.4 years (range 1-16 (SD 1.7)).

A total of 195 (87.1\%) participants completed the "wellbeing in adversity" item at baseline. Completers had a higher mean MMSE score than non-completers (16.7 (SD 7.0) vs 1.5 (SD 2.5); $\mathrm{t}=22.4, \mathrm{p}<0.001)$. The two groups did not differ significantly with regard to mean age (completers 81.1 (7.5) vs. 80.9 (7.4)) or depression score (4.6 (4.8) vs 3.8 (5.3)).

Mean "wellbeing in adversity" score was 2.9 (SD 0.8). Eleven participants (4.9\%) rated it as "poor," 44 (19.6\%) as "fair," 97 (43.3\%) as "good" and $43(19.2 \%)$ as "excellent."

\section{Participants at $\mathbf{1 8}$ months}

At the 18 month follow-up, 48 patients had died, eight refused and one had moved away; 167/176 (94.9\%) of those still alive participated in the 18 month follow-up, of whom 122 (73.1\%) participants completed the "wellbeing in adversity" ratings. Completers had a higher mean MMSE score than noncompleters (19.3 (SD 5.9), 95\% CI 18.2 to 20.3 vs 9.3 (SD 7.6),
95\% CI 7.8 to $10.8 ; \mathrm{t}=10.9, \mathrm{p}<0.001)$, and they had also deteriorated less on MMSE scores ( -2.5 (SD 4.5) vs -4.6 (SD 5.5); Mann-Whitney U test 2130.0, $\mathrm{p}<0.05)$. The two groups did not differ significantly with regard to mean age (completers 81.1 (SD 7), 95\% CI 79.2 to 81.8 vs 80.9 (SD 7.4), $95 \%$ CI 80.3 to 83.3), mental health (completers 76.7 (SD 19.1), 95\% CI 73.3 to 80.1 vs 71.3 (SD 21.5), 95\% CI 66.1 to 76.5 ) or depression score (4.6 (SD 4.8), 95\% CI 3.1 to 4.5 vs 3.8 (SD 5.3), 95\% CI 4.4 to 7.3).

\section{Univariate analyses}

Table 1 shows the descriptive statistics for all variables and correlations between the postulated baseline predictors and wellbeing in adversity at 18 months. Social relationships, both indicators of mental health, health perception, $\mathrm{ADL}$ and baseline wellbeing in adversity, were significant correlates of wellbeing in adversity at 18 months on univariate analysis.

Table 2 shows the correlations among the baseline factors significantly associated with wellbeing in adversity at 18 months. All were significantly correlated with each other, with the exception of ADL score, which did not correlate with mental health or health perception, and global dementia severity, which did not correlate with health perception or baseline wellbeing in adversity.

\section{Stability of "wellbeing in adversity"}

Of the 122 participants who completed the "wellbeing in adversity" measure at both baseline and 18 months, 77 (63.1\%) did not change their rating over time; 24 (19.7\%) rated it higher (four improved by 2 points on the measure and 20 improved by 1 point); 21 (17.2\%) scored lower (one by 3 points, three by 2 points and 17 by 1 point). Mean "wellbeing in adversity" scores did not change significantly over time (mean 2.97 (SD 0.738 ) vs 2.98 (0.738); $t=0.232$, NS). The correlation value between wellbeing in adversity scores at baseline and 18 months is presented in table 1 .

\section{Multivariate analyses (table 3)}

On step 1 of our linear regression, we entered baseline ADL and health perception, and only health perception predicted wellbeing in adversity at 18 months. In step 2, we added social functioning; only social functioning was then a significant predictor. In step 3, we entered mental health score. Only mental health was a significant predictor. In step 4, we added baseline wellbeing in adversity. Baseline wellbeing in adversity and mental health score were the only significant predictors in the final model.

\section{Structural equation modelling}

To test our first hypothesis, we entered global dementia, health perception, social relationships and mental health into a path

Table 2 Inter-correlations of baseline factors (including baseline wellbeing score) which were significantly correlated with wellbeing at 18 months (Pearson's correlation coefficient)

\begin{tabular}{|c|c|c|c|c|c|c|}
\hline Variable & $\begin{array}{l}\text { Activities } \\
\text { of daily } \\
\text { living }\end{array}$ & $\begin{array}{l}\text { Health } \\
\text { perception }\end{array}$ & $\begin{array}{l}\text { Mental } \\
\text { health }\end{array}$ & $\begin{array}{l}\text { Social } \\
\text { relationship }\end{array}$ & $\begin{array}{l}\text { Global } \\
\text { dementia } \\
\text { severity }\end{array}$ & $\begin{array}{l}\text { Wellbeing } \\
\text { in adversity } \\
\text { (baseline) }\end{array}$ \\
\hline Activities of daily living & - & -0.15 & 0.16 & $0.30 * *$ & $-0.81^{* * *}$ & $0.22^{*}$ \\
\hline Health perception & & - & $0.44^{* * *}$ & $0.28 * *$ & -0.11 & $0.48^{* * *}$ \\
\hline Mental health & & & - & $0.43^{* * *}$ & $-0.20^{* *}$ & $0.45^{* * *}$ \\
\hline Social relationships & & & & - & $-0.40 * * *$ & $0.50 * * *$ \\
\hline Global dementia severity & & & & & - & -0.13 \\
\hline
\end{tabular}

${ }^{*}$ Correlation significant at the 0.05 level; ${ }^{* *}$ correlation significant at the 0.01 level; ${ }^{* * *}$ correlation significant at the 0.001 level 
Table 3 Stepwise linear regression with "wellbeing in adversity" at 18 months as the dependent variable

\begin{tabular}{llccl}
\hline Model & & $\begin{array}{l}\text { Standardised } \\
\text { coefficients } \\
\text { beta }\end{array}$ & $\mathbf{t}$ & p Value \\
\hline 1 & Health perception & 0.229 & 2.458 & 0.016 \\
2 & Health perception & 0.154 & 1.639 & 0.104 \\
& Social relationships & 0.266 & 2.818 & 0.006 \\
3 & Health perception & 0.053 & 0.545 & 0.587 \\
& Social relationships & 0.166 & 1.692 & 0.094 \\
& Mental health & 0.297 & 2.845 & 0.005 \\
4 & Health perception & -0.029 & -0.286 & 0.776 \\
& Social relationships & 0.069 & 0.666 & 0.507 \\
& Mental health & 0.274 & 2.675 & 0.009 \\
& Wellbeing in adversity (baseline) & 0.258 & 2.386 & 0.019 \\
\hline $\mathrm{r}^{2}=0.22$. & & &
\end{tabular}

analysis to generate a model to predict wellbeing in adversity 18 months later. Figure 1 shows the relationships between the significant baseline predictors of wellbeing in adversity at 18 months (excluding baseline wellbeing in adversity). A structural equation model $\left(\chi^{2}=3.6, \mathrm{p}=0.31, \mathrm{df}=3\right)$ based on these results had a NFI of 0.98 , indicating a well fitting model. Less severe dementia and better health perception predicted fewer mental health problems and social relationships, but were not direct predictors of wellbeing in adversity at 18 months directly. Better mental health and social relationships were strong predictors of wellbeing.

\section{DISCUSSION}

This is the first longitudinal study to consider independent determinants of wellbeing in dementia related adversity. Subjective wellbeing in patients with dementia is, we believe, an important outcome to consider. Our choice of factors to analyse was informed by the literature relating to self-rated QoL in dementia. We confirmed our hypothesis that future wellbeing was directly predicted by mental health (anxiety and depression) and social relationships rather than by either global dementia severity or general health. In contrast with earlier literature that defined "successful ageing" as lack of illness, our measure of "wellbeing in adversity" was not related to age. General health perception was associated with subsequent wellbeing in adversity on univariate analyses, but results of our multivariate analysis and SEM indicate that this relationship is mediated by mental health and possibly by social relationships. They were the only significant independent predictors of "wellbeing in adversity" in a well fitting SEM. The effect of mental health on wellbeing was partially accounted for by its effect on social relationships. We also confirmed our second hypothesis that wellbeing in adversity would be stable over time which may reflect an individual's underlying resilience. As in an earlier study ${ }^{11}$ we found no mean change in self-reported wellbeing ratings longitudinally, this reliability also suggests that the ratings are meaningful despite the raters having dementia. Nonetheless, there was some individual changeboth increases and decreases-in wellbeing over time. In contrast, cognition in this cohort rarely improved over time.

The high mean score of wellbeing in dementia adversity is in line with other studies illustrating the "disability paradox" and are neither because people with dementia are cognitively incapable of rating their wellbeing nor because of their lack of insight into it. We believe our findings are clinically useful as they carry the positive message that, in general, people with dementia feel that they are living successfully. In addition, by demonstrating for the first time that mental health and social relationships are the key predictors of future wellbeing in dementia, our study suggests that preventative interventions may be a future possibility. There is considerable evidence that depression is often overlooked and untreated in older people with dementia (both in the community and particularly in institutional settings). The important clinical implications of this study are that actively managing depression and anxiety may not only relieve current distress but also have long term benefits for wellbeing.

Our findings are also similar to another recent study which found that social factors and mental health were cross sectional predictors of QoL in older people with physical illness. ${ }^{27}$ This suggests that these factors may predict successful ageing across illnesses.

We employed validated measures administered by trained raters who were not aware of the hypotheses tested in this paper. In addition, the population was purposively selected to be representative of people with $\mathrm{AD}$ and was from urban, suburban and semi-rural areas, and as such the findings should be generalisable to similar populations. They are however not generalisable to those with the most severe dementia, or those who deteriorated most cognitively, who could not complete the measure or died during the study. As those who did not complete the measure deteriorated more on MMSE scores, we did a post hoc analysis, and found that change in MMSE score did not correlate with change in wellbeing in the participants. Subjective wellbeing in patients with dementia is interesting but a potentially difficult concept to measure. While people with dementia, who have problems with communication and memory, might not be able to meaningfully answer such questions, there is evidence that they are able to give acceptably valid and reliable answers. ${ }^{10-12}$ In addition, we have shown that wellbeing in adversity is related to social and mental health, but we did not measure inherent resilience and this may be why our model did not account for more of the variance.
Figure 1 Structural equation model testing the hypothesis that social relationships and mental health predicted wellbeing in adversity 18 months later (numbers denote standardised regression coefficients; e = latent error).

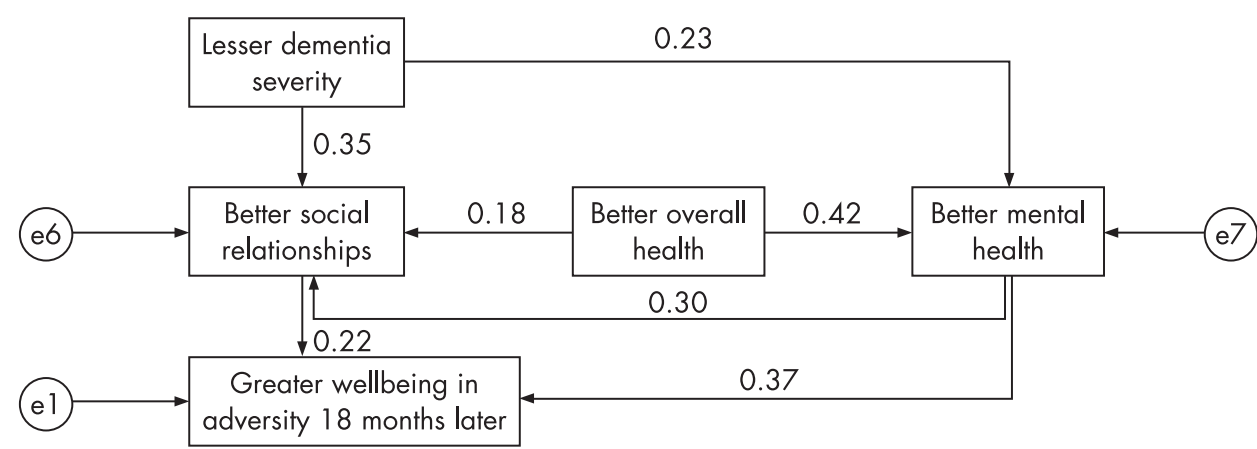


Our approach could be applied to exploring "ageing in adversity" among populations with other long term conditions or indeed those who care for them. These studies would benefit from including measures of resilience. Perhaps most importantly, they could guide interventions studies to promote successful ageing in these populations.

As the number of people living in the community with dementia increases, it is ever more important to improve understanding of their QoL and, in particular, to the role played_or not-by their illness. We hope to have made a distinctive contribution to an emerging discourse on how to integrate literature on illness with that on quality of life and "successful" living in older age. This study helps to broaden the generally narrow conceptualisation of "successful aging" beyond its emphasis on avoiding health related impairments to encompass a subjective sense of overall wellbeing regardless of living with impairments and to further understand the contributors of this important outcome in a population of people with dementia.

Acknowledgements: We would like to thank all the people with $A D$ and their families, friends and other carers who participated in the study. We would also like to thank all the consultant psychiatrists and community mental health teams along with the staff of the nursing and residential homes who provided us with support and help.

Funding: Funding was received from Lundbeck SA.

Competing interests: None.

Ethics approval: The local research ethics committees gave ethics approval for the study.

\section{REFERENCES}

1. Depp CA, Jeste DV. Definitions and predictors of successful aging: a comprehensive review of larger quantitative studies. Am J Geriatr Psychiatry 2006;14:6-20.

2. Ready RE, Ott B, Grace J. Patient versus informant perspecives of quality of life in mild cognitive impairment and Alzheimer's disease. Int J Geriatr Psychiatry 2004;19:256-65

3. Albrecht GL, Devlieger PJ. The disability paradox: high quality of life against all odds. Soc Sci Med 1999:48:977-88.

4. Vogel A, Mortensen EL, Hasselbalch SG, et al. Patient versus informant reported quality of life in the earliest phases of Alzheimer's disease. Int J Geriatr Psychiatry 2006;21:1132-8.

5. Ready R, Ott B. Quality of life measures for dementia. Health Qual Life Outcomes 2003:1:11.

6. Merchant C, Hope KW. The quality of life in Alzheimer's disease scale: direct assessment of people with cognitive impairment. J Clin Nurs 2004;13:105-10.

7. Covinsky KE, Wu AW, Landefeld CS, et al. Health status versus quality of life in older patients: does the distinction matter? Am J Med 1999;106:435-40

8. Sloane PD, Zimmerman S, Williams CS, et al. Evaluating the quality of life of longterm care residents with dementia. Gerontologist 2005;45:37-49.

9. Katsuno T. Personal spirituality of persons with early-stage dementia: is it related to perceived quality of life? Dementia 2003;2:315-35

10. Hoe J, Katona $\mathrm{C}$, Roch $\mathrm{B}$, et al. Use of the QOL-AD for measuring quality of life in people with severe dementia-the LASER-AD study. Age Ageing 2005;34:130-5.

11. Selwood A, Thorgrimsen L, Orrell M. Quality of life in dementia - a one-year followup study. Int J Geriatr Psychiatry 2005;20:232-7.

12. Thorgrimsen L, Selwood A, Spector A, et al. Whose quality of life is it anyway? The validity and reliability of the quality of life-Alzheimer's disease (0OL-AD) scale. Alzheimer Dis Assoc Disord 2003;17:201-8.

13. Scocco $\mathbf{P}$, Fantoni $G$, Caon F. Role of depressive and cognitive status in self-reported evaluation of quality of life in older people: comparing proxy and physician perspectives. Age Ageing 2006;35:166-71.

14. James BD, Xie SX, Karlawish JHT. How do patients with Alzheimer disease rate their overall quality of life? Am J Geriatr Psychiatry 2005;13:484-90.
15. Cahill S, Begley E, Topo P, et al. "I know where this is going and I know it won't go back": hearing the individual's voice in dementia quality of life assessments. Dementia 2004;3:313-30.

16. Fuh J-L, Wang S-J. Assessing quality of life in Taiwanese patients with Alzheimer's disease. Int J Geriatr Psychiatry 2006;21:103-7.

17. Hoe J, Hancock G, Livingston G, et al. Quality of life of people with dementia in residential care homes. Br J Psychiatry 2006;188:460-4.

18. Logsdon RG, Gibbons LE, McCurry SM, et al. Assessing quality of life in older adults with cognitive impairment. Psychosom Med 2002:64:510-19.

19. Logsdon RG, Gibbons LE, McCurry SM, et al. Quality of life in Alzheimer's disease: patient and caregiver reports. J Ment Health Aging 1999;5:21-32.

20. Shin HY. A preliminary study on the Korean version of quality of life-Alzheimer's disease (OOL-AD) scale in community-dwelling elderly with dementia. J Prev Med Pub Health 2006:39:243-8.

21. Snow AL, Dani R, Souchek J, et al. Comorbid psychosocial symptoms and quality of life in patients with dementia. Am J Geriatr Psychiatry 2005;13:393-401.

22. Selai CE, Trimble MR, Rosser MN, et al. Assessing quality of life in dementia: preliminary psychometric testing of the quality of life assessment schedule (QOLAS) Neuropsychol Rehabil 2001;11:219-43.

23. Wlodarczyk $\mathbf{J H}$, Brodaty $\mathrm{H}$, Hawthorne $\mathrm{G}$. The relationship between quality of life, mini-mental state examination, and the instrumental activities of daily living in patients with Alzheimer's disease. Arch Gerontol Geriatr 2004:39:25-33.

24. Matsui T, Nakaaki S, Murata Y, et al. Determinants of the quality of life Alzheimer's disease patients as assessed by the Japanese version of the quality of lifeAlzheimer's disease scale. Dement Geriatr Cogn Disord 2006;21:182-91.

25. Ready RE, Ott BR, Grace J, et al. The Cornell-Brown scale for quality of life in dementia. Alzheimer Dis Assoc Disord 2002;16:109-15.

26. Edelman P, Fulton BR, Kuhn D, et al. A comparison of three methods of measuring dementia-specific quality of life: perspectives of residents, staff, and observers. Gerontologist 2005;45:27-36.

27. Bowling A, Seetai S, Morris R, et al. Quality of life among older people with poor functioning. The influence of perceived control over life. Age Ageing 2007:36:310-15.

28. Regan C, Katona C, Walker Z, et al. Relationship of vascular risk to the progression of Alzheimer disease. Neurology 2006;67:1357-62.

29. Livingston G, Walker AE, Katona CLE, et al. Antipsychotics and cognitive decline in Alzheimer's disease: the LASER-Alzheimer's disease longitudinal study. J Neurol Neurosurg Psychiatry 2007;78:25-9.

30. Fratiglioni L. Epidemiology. In: Wimo A, Jonsson B, Karlsson G, et al, eds. Health economics of dementia. New York: John Wiley \& Sons, 1999:13-31.

31. McKhann G, Drachman D, Folstein, et al. Clinical diagnosis of Alzheimer's disease: report of the NINCDS-ADRDA work group under the auspices of Department of Health and Human Services Task Force on Alzheimer's Disease. Neurology 1984:34:939-44.

32. Folstein MF, Folstein SE, McHugh PR. Mini-Mental State. A practical method for grading the cognitive state of patients for the clinician. J Psychiatr Res 1975;12:18998.

33. Cummings JL, Mega M, Gray K, et al. The neuropsychiatric inventory: comprehensive assessment of psychopathology in dementia. Neurology 1994:44:2308.

34. Galasko D, Bennett D, Sano M, et al. An inventory to assess activities of daily living for clinical trials in Alzheimer's disease. The Alzheimer's disease cooperative study. Alzheimer Dis Assoc Disord 1997;11(Suppl 2):33-9.

35. Alexopoulos GS, Abrams RC, Young RC, et al. Cornell scale for depression in dementia. Biol Psychiatry 1988;23:271-84.

36. Schneider LS, Olin JT, Doody RS, et al. Validity and reliability of the Alzheimer's disease cooperative study — clinical global impression of change. The Alzheimer disease cooperative study. Alzheimer Dis Assoc Disord 1997;11(Suppl 2):22-32.

37. Radosevich D, Pruitt M. Twelve-item health status questionnaire: HSQ-12 version 2.0. Bloomington: Heath Outcomes Institute, 1995.

38. Petit T, Livingston G, Manela M, et al. Validation and normative data of health status measures in older people: the Islington study. Int J Geriatr Psychiatry 2001;16:106170.

39. Miles J, Shevlin M. Applying regression and correlation: a guide for students and researchers. London: Sage Publications, 2001.

40. Baron RM, Kenny DA. The moderator-mediator variable distinction in social psychological research: conceptual, strategic, and statistical considerations. J Pers Soc Psychol 1986;51:1173-82

41. Bentler PM, Bonett DG. Significance tests and goodness of fit in the analysis of covariance structures. Psychol Bull 1980;88:588-606. 